\title{
Dynamic Takagi-Sugeno Model for the Control of Ultrasonic Motor
}

\author{
Shi Jingzhuo, ${ }^{1}$ Lv Lin, ${ }^{1}$ and Zhang $\mathbf{Y u}^{2}$ \\ ${ }^{1}$ Department of Automation, Henan University of Science and Technology, Luoyang 471003, China \\ ${ }^{2}$ Department of Automation, Southeast University, Nanjing 210096, China
}

Correspondence should be addressed to Shi Jingzhuo, sjznew@163.com

Received 13 May 2011; Revised 7 July 2011; Accepted 22 July 2011

Academic Editor: Mohamed Zribi

Copyright (C) 2011 Shi Jingzhuo et al. This is an open access article distributed under the Creative Commons Attribution License, which permits unrestricted use, distribution, and reproduction in any medium, provided the original work is properly cited.

\begin{abstract}
Model of ultrasonic motor is the foundation of the design of ultrasonic motor's speed and position controller. A two-input and one-output dynamic Takagi-Sugeno model of ultrasonic motor driving system is worked out using fuzzy reasoning modeling method in this paper. Many fuzzy reasoning modeling methods are sensitive to the initial values and easy to fall into local minimum, and have a large amount of calculation. In order to overcome these defects, equalized universe method is used in this paper to get clusters centers and obtain fuzzy clustering membership functions, and then, the unknown parameters of the conclusions of fuzzy rules are identified using least-square method. Different experimental data that are tested with different operational conditions are used to examine the validity of the fuzzy model. Comparison between experimental data and calculated data of the model indicates that the model can well describe the nonlinear characteristics among the frequency, amplitude of driving voltage and rotating speed. The proposed fuzzy model can be used to analyze the performance of ultrasonic motor driving system, and also can be used to design the speed and position controller of ultrasonic motor.
\end{abstract}

\section{Introduction}

The model of ultrasonic motor system is the foundation of analyzing ultrasonic motor's performance and is also an important premise of designing motor controller and trying to improve the control performance. The energy conversion processes of ultrasonic motor have several stages $[1,2]$. The first is the electromechanical energy conversion using the inverse piezoelectric effect of piezoelectric material on the stator. And then, the motion of particles on the surface of stator is transformed into rotary motion of rotor by the friction on the contact surface. Because of the nonlinear and time-varying operation of piezoelectric material and friction, the strong nonlinear characteristics and complex coupling relations of ultrasonic motor are brought out by the complicated energy conversion processes and nonlinearity of material [2]. Because of these reasons, the accurate theoretical model of ultrasonic motor is difficult to obtain.

Modeling of ultrasonic motor can adopt different methods. The equivalent circuit of ultrasonic motor is a kind of model that can be used to analyze the basic characteristics of ultrasonic motors [3]. Because the electrical circuit is more familiar to us than the piezoelectric material and friction, the equivalent circuit was widely used in the early stage of the study on ultrasonic motor. But the equivalent circuit is a rude modeling method of the dynamic characteristics of ultrasonic motor. On the other hand, this type of model cannot be used to design motor's controller directly. From the perspective of being suitable for control applications, the study gets more and more attention on the control modeling method of ultrasonic motor system. Usually, for the purpose of online realization and minimizing the amount of online calculation, the control model should be relatively simple and show the main aspects of nonlinear control of ultrasonic motor system. Due to the limitations of theoretical modeling, control modeling usually adopts identification method based on the tested data [4-11]. According to the different identification methods, the forms of model can be the transfer function, differential equation, neural network, and so forth.

In recent years, fuzzy modeling method based on fuzzy reasoning is gradually arisen. The same as in the neural network model, fuzzy model is also based on experimental 
data, easy to show the nonlinear information. People's fuzzy thinking process is simulated by fuzzy reasoning. So, fuzzy model is more easily mixed and makes use of people's relevant experience knowledge. This is different from other methods. The complexity of fuzzy model is relatively low. So, another effective way is provided for the modeling of nonlinear complex system. The fuzzy modeling method is rarely used in the field of motor. The fuzzy method is mostly used to realize rotating speed and position control [1214]. Phase difference is used as the controlled variable in [15], and a kind of fuzzy Hammerstein model is worked out. This model is made up of steady nonlinear part and dynamic linear transfer function. According to the experimental measurements, the relationship between steady rotating speed and driving frequency is realized by fuzzy steady model.

This paper works out the appropriate fuzzy modeling method to obtain the dynamic model of ultrasonic motor system. Equalized universe method is used to get clusters centers and obtain fuzzy clustering membership functions. And then least-square-method is used to identify the unknown parameters of the conclusions of fuzzy rules. The two-input and one-output Takagi-Sugeno model of ultrasonic motor system is established, and the model can well show the nonlinear dynamic relationship among the amplitude of driving voltage, frequency, and rotating speed.

\section{Experimental Data Used for Modeling}

The block diagram of experimental system is shown in Figure 1. An optoelectric encoder, $E$, is used to measure the rotating speed of ultrasonic motor. And the voltage amplitude controllers of phases $\mathrm{A}$ and $\mathrm{B}$ are designed to make the voltage amplitude of the two phases equal to the given value by adjusting the duty ratio of PWM signals. In the figure, Nref and Uref are the given value of rotating speed and amplitude of driving voltage, respectively.

Supply voltage of the circuit is DC12V. Phase-shift PWM method is used in the circuit to control the H-bridge driver as shown in Figure 2. In Figure 2, Q1 $\sim 8$ are power MOSFETs, T1 and T2 are transformers, and $L 1$ and $L 2$ are series compensating inductances. Figure 3 is the equivalent circuit of ultrasonic motor's stator. $C_{d}$ is the capacitance due to the piezoelectric element's dielectric properties called the "tank capacitance." $R_{m}, C_{m}$, and $L_{m}$ are known as the motional impedance, and they are combined in the form $\left(R_{m}+1 / j \omega C_{m}+j \omega L_{m}\right)$. Therefore, from the viewpoint of the driving circuit, the ultrasonic motor is a kind of capacitive load. To compensate this capacitive load, series compensating inductances $L 1$ and $L 2$ are used here to increase the driving performance.

PWM1 PWM8 are the PWM control signals for Q1 Q8. The traveling ultrasonic motor used here is the commercial used ultrasonic motor, Shinsei USR60. The principal specification of USR60 motor is shown in Table 1. In the control circuit, DSP is used to implement the control strategies shown in Figure 1, which is type DSP56F801.
TABLE 1: Specification of USR60 motor.

\begin{tabular}{lc}
\hline Item & Value \\
\hline Driving voltage & $130 \mathrm{Vrms}$ \\
Rated torque & $0.5 \mathrm{Nm}$ \\
Rated output power & $5.0 \mathrm{~W}$ \\
Rated rotating speed & $100 \mathrm{r} / \mathrm{min}$ \\
Max. torque & $1.0 \mathrm{Nm}$ \\
Temperature range & $-10 \sim+55^{\circ} \mathrm{C}$ \\
Weight & $260 \mathrm{~g}$ \\
\hline
\end{tabular}

The CPLD is used to produce the phase-shift PWM control signals PWM1 PWM8 for H-bridge.

The controllable variables that can be used for rotating speed control are the amplitude of driving voltage, frequency, and phase difference of the two-phase voltages. The theoretical analyses and related experimental studies show that if the phase difference of two-phase voltages is set to $\pm 90^{\circ}$, better operation performance can be maintained and the energy conversion efficiency of ultrasonic motor is higher. In this paper, the phase difference of two-phase voltages is set to $\pm 90^{\circ}$. The remainder controllable variables, amplitude and frequency of driving voltage can be adjusted to realize the aim of speed control. Therefore, the dynamic data, which contain the amplitude of driving voltage, frequency, and speed, should be measured in the experiments. A dynamic fuzzy model, which uses the amplitude of driving voltage and frequency as input and rotating speed as output, is established.

Experiment process is designed as follows. The step response of rotating speed is measured by setting Nref. The amplitude of driving voltage is changed by adjusting Uref. With the change of voltage amplitude, the frequency will be changed by speed closed-loop controller in order to maintain the rotating speed at Nref. Simultaneously, the dynamic change processes of Uref, frequency and rotating speed are recorded. A group of data is shown in Figure 4. The adjustment method of Uref and value of Nref are changed, respectively. The above processes are repeated. Many groups of data can be obtained for fuzzy modeling.

\section{Fuzzy Modeling Method of Ultrasonic Motor}

The basic structure of fuzzy model is shown in Figure 5. In fuzzy models, T-S model which can approximate to any nonlinear system is a kind of typical dynamic model. The form of T-S model is as follows. The premise section is fuzzy, and the conclusion section is an exact value. So, the defuzzification is not necessary in T-S model

$$
\begin{aligned}
& R_{i}: \text { If }\left(x_{1} \text { is } A_{i 1}\right), \ldots,\left(x_{k} \text { is } A_{i k}\right), \ldots,\left(x_{r} \text { is } A_{i r}\right), \\
& \text { then } y_{i}=a_{i 0}+a_{i 1} x_{1}+\cdots+a_{i k} x_{k}+\cdots+a_{i r} x_{r} .
\end{aligned}
$$

Here, $R_{i}$ represents the $i$ th fuzzy rule, $x_{k}$ is the $k$ th input variable, $A_{i k}$ is one of the fuzzy subsets of $x_{k}, y_{i}$ is the output of the $i$ th rule $R_{i}$, and $a_{i k}$ is the unknown parameter of the rule's conclusion, $k=0, \ldots, r$. 


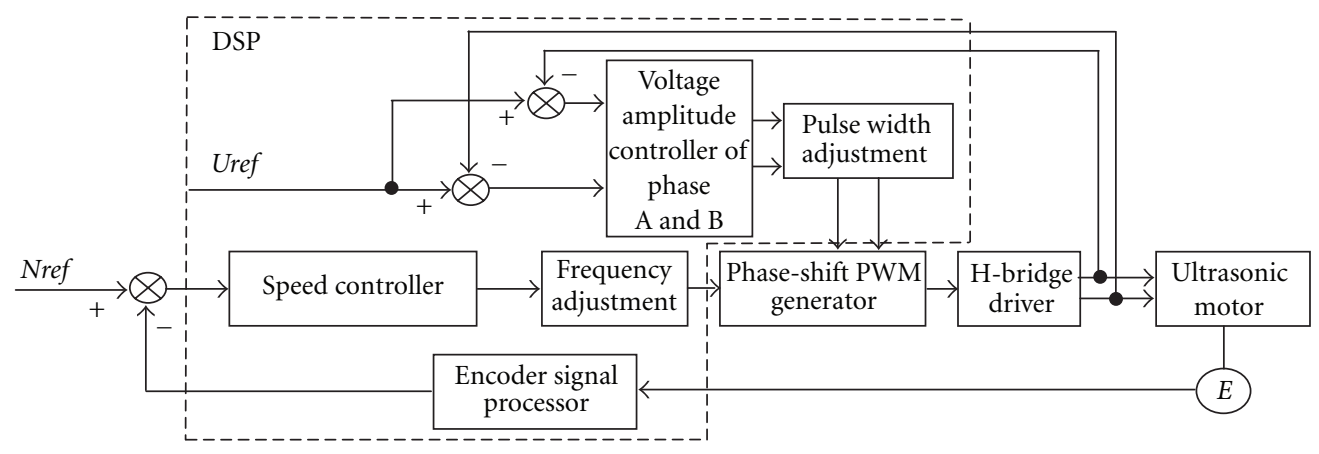

FIGURE 1: Structure of the experimental system for speed control.

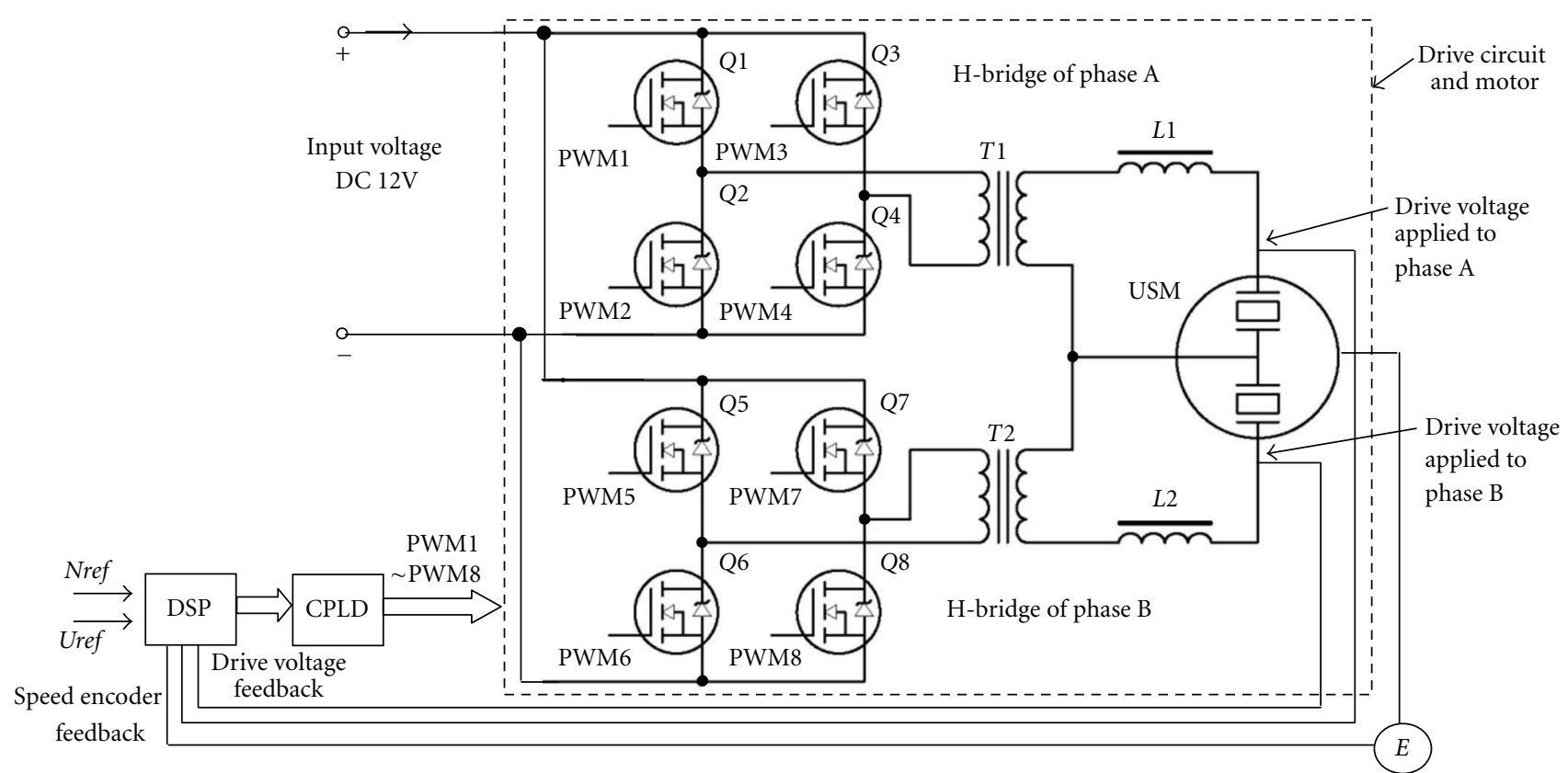

FIGURE 2: Structure of the driving and control circuit.

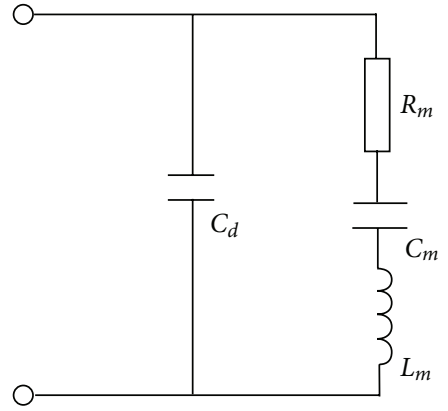

FIgURE 3: Equivalent circuit of ultrasonic motor's stator.

According to the tested input and output data, structure identification and parameter identification are required in establishing the T-S model of ultrasonic motor system. Determining the appropriate model structure is the basis of parameter identification. The first step of structure identification is to confirm the input variables and the

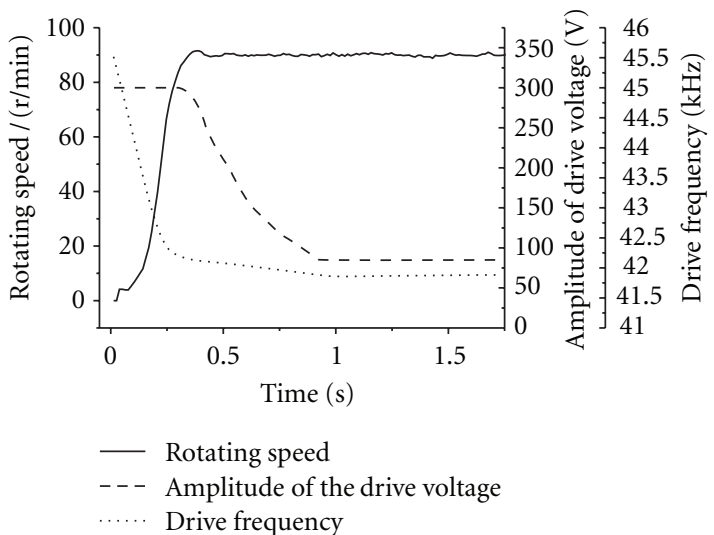

Figure 4: Tested data of speed control.

input space. This paper adopts equalized universe method to divide the fuzzy input space. First, set a clustering number which is the number of membership functions and rules. 


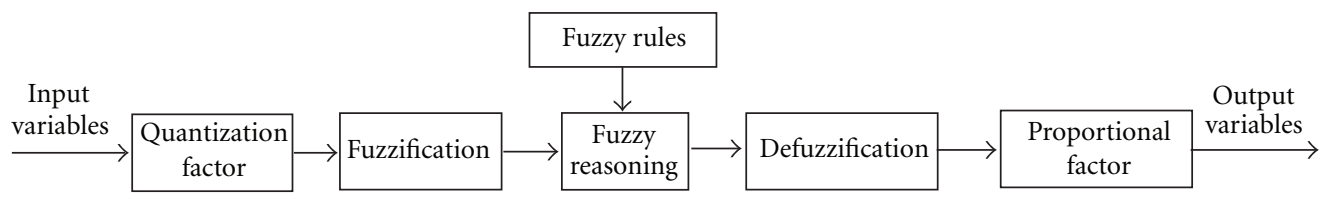

FIgURE 5: Basic structure of fuzzy model.

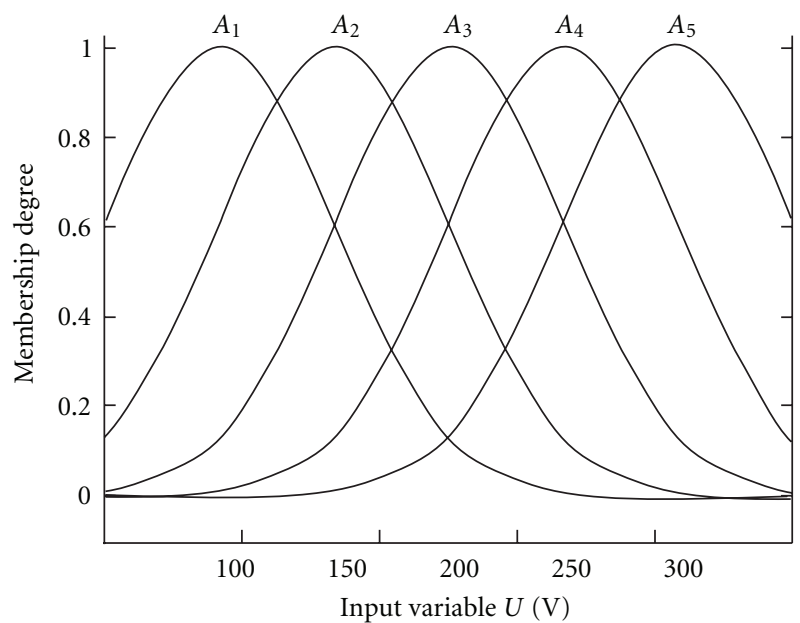

FIgURE 6: Membership functions of $U$.

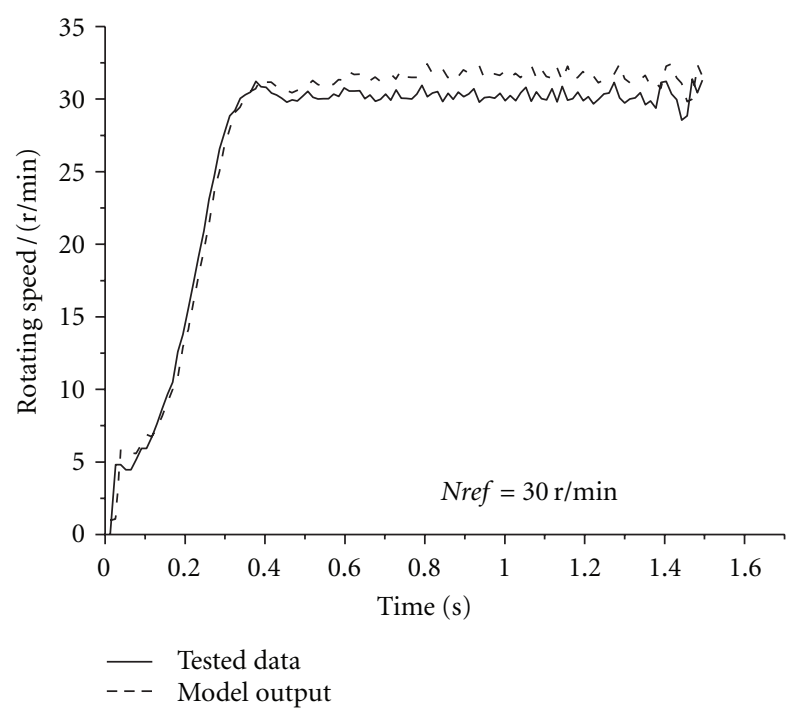

Figure 7: Comparison between model output and tested data $($ Nref $=30 \mathrm{r} / \mathrm{min})$.

Secondly, divide the input space. Equalized universe method is used to determine the characters of Gaussian membership function, such as value of the center and width. The structure identification of T-S model is completed. The unknown parameters of conclusion section of T-S model can be determined using the least square method. After the model is established, the validation calculation should be carried on. If the error of model output is too large, the number of rules can be increased to improve the precision.

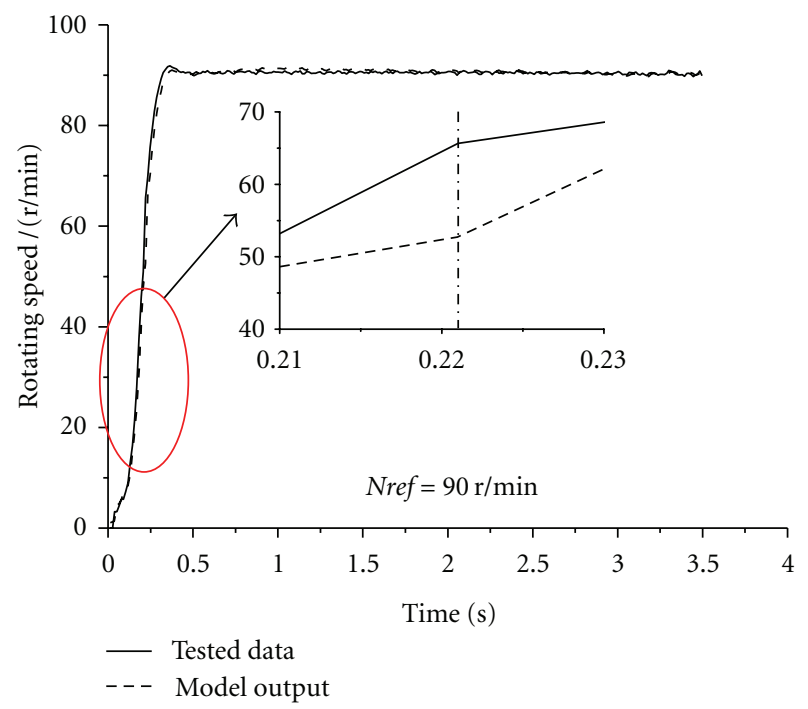

Figure 8: Comparison between model output and tested data $($ Nref $=90 \mathrm{r} / \mathrm{min})$.

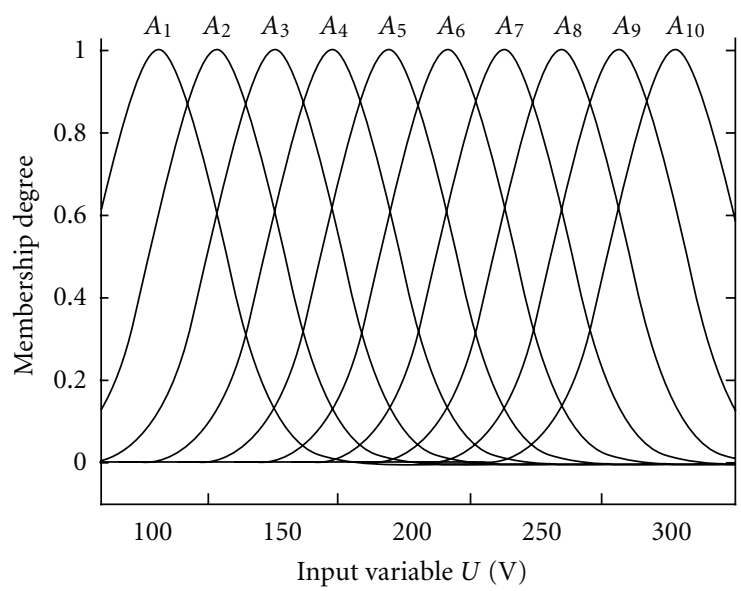

FIgURE 9: Membership functions of $U$.

The steps of this fuzzy modeling method can be summarized as follows.

(1) Determine the input variables, the number $p$ of input variables, and the number $c$ of clusters.

(2) According to the analysis of tested data, determine the corresponding domain space $\left[\mathrm{M}^{-}, \mathrm{M}^{+}\right]$of driving voltage amplitude, frequency, and rotating speed. 


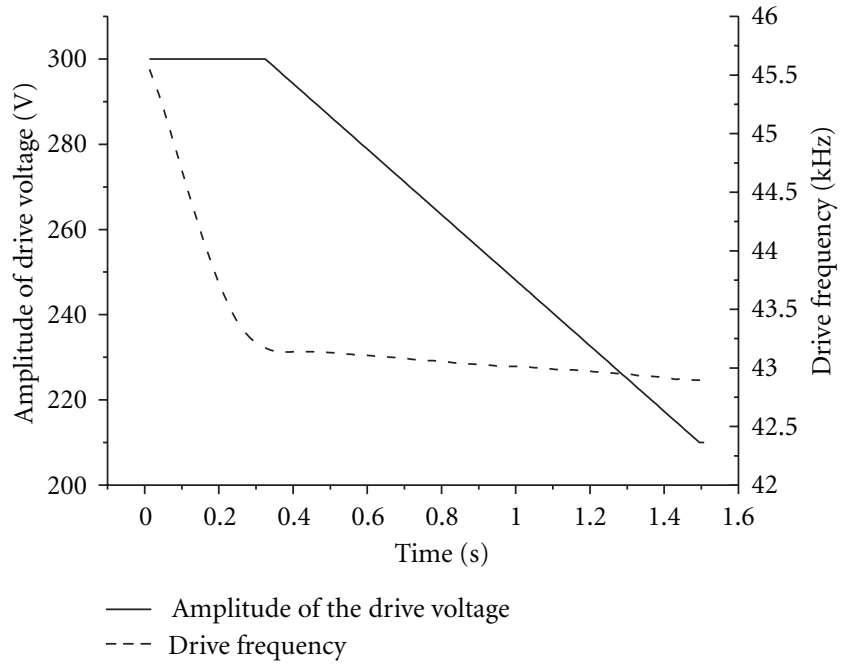

(a) The curve of drive voltage and frequency

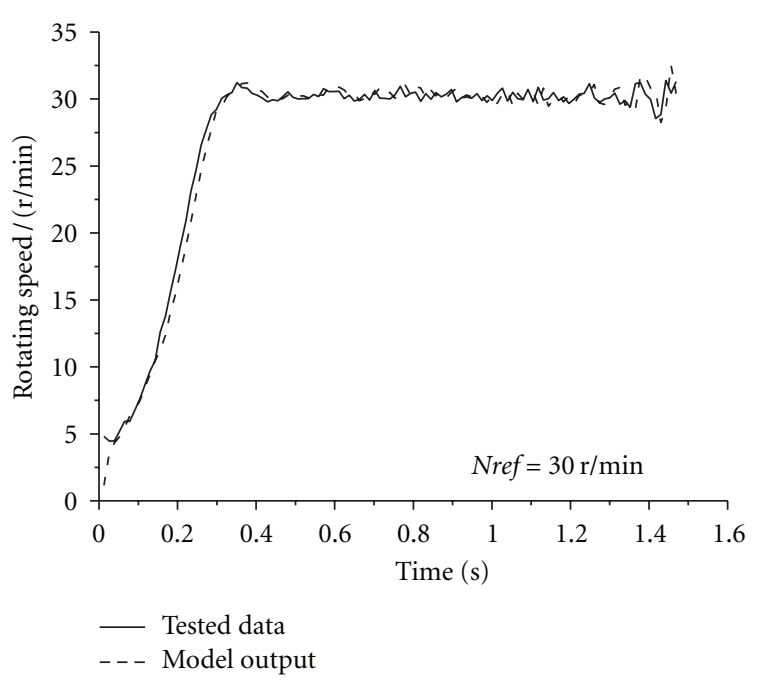

(b) Comparison between model output and tested data

Figure 10: Step response comparison between model output and tested data (Nref $=30 \mathrm{r} / \mathrm{min}$, no load).

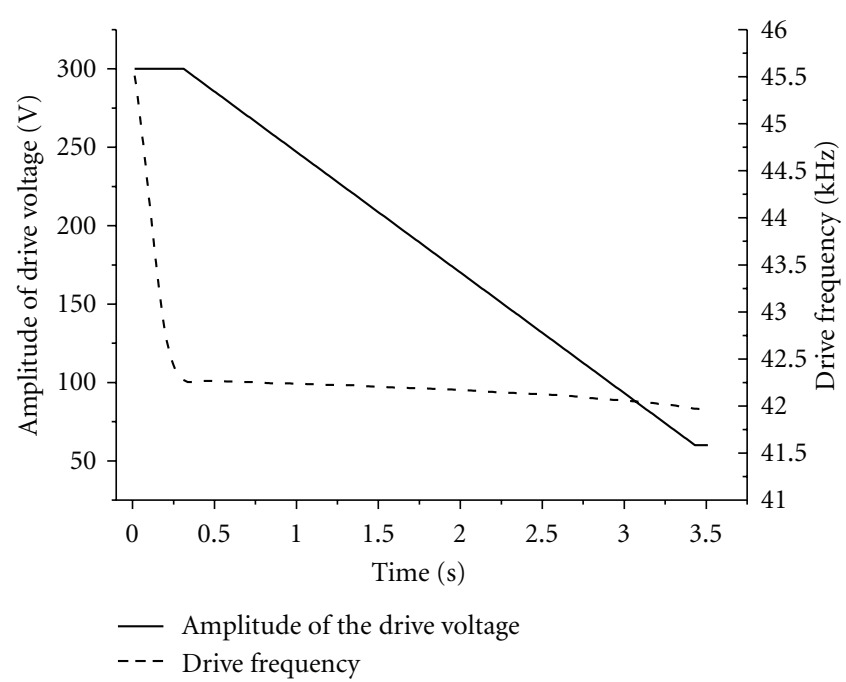

(a) The curve of drive voltage and frequency

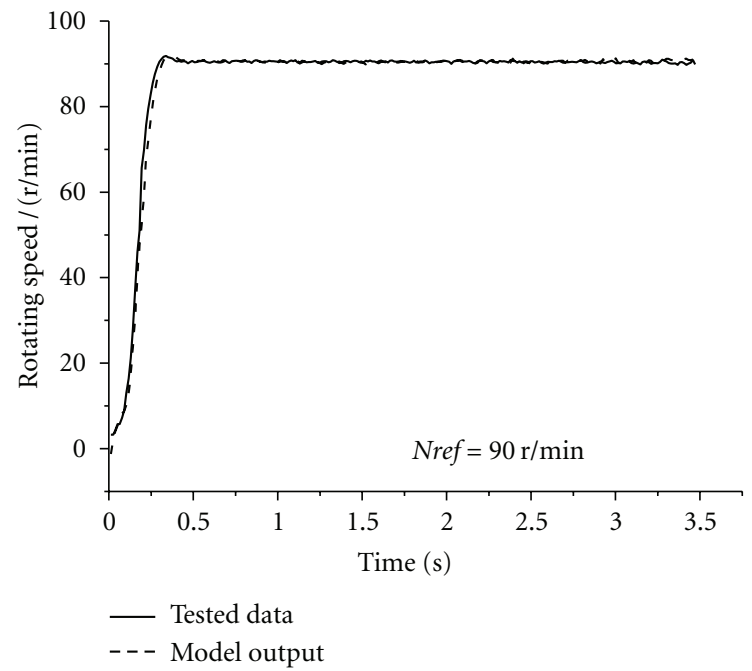

(b) Comparison between model output and tested data

FIGURE 11: Step response comparison between model output and tested data (Nref $=90 \mathrm{r} / \mathrm{min}$, no load).

(3) Determine the centers $\overline{V_{i}}$ and widths $h_{i}$ of Gaussian membership functions using equalized universe method

$$
\begin{gathered}
\overline{V_{i}}=M^{-}+\frac{M^{+}-M^{-}}{c+1} i, \quad(i=1,2, \ldots, c), \\
h_{i}=\frac{M^{+}-M^{-}}{c+1} .
\end{gathered}
$$

(4) Calculate the distance $d_{i k}$ between the each data point $x_{k}$ and clustering center $\overline{V_{i}}$

$$
d_{i k}=\left\|x_{k}-v_{i}\right\|, \quad(k=1,2, \ldots, n) .
$$

(5) Calculate the membership degree of modeling data

$$
\mu_{i k}=\frac{1}{\sum_{j=1}^{c}\left(d_{i k} / d_{j k}\right)^{2 /(m-1)}} .
$$

Here, $m$ is a constant, usually set $m=2$.

(6) The unknown parameters of conclusion section of T$S$ model are obtained by the least-square method.

(7) If the precision of model is not satisfactory after validation calculation, change the number $c$ of clusters and/or the number $p$ of input variables, and go to step (2). 


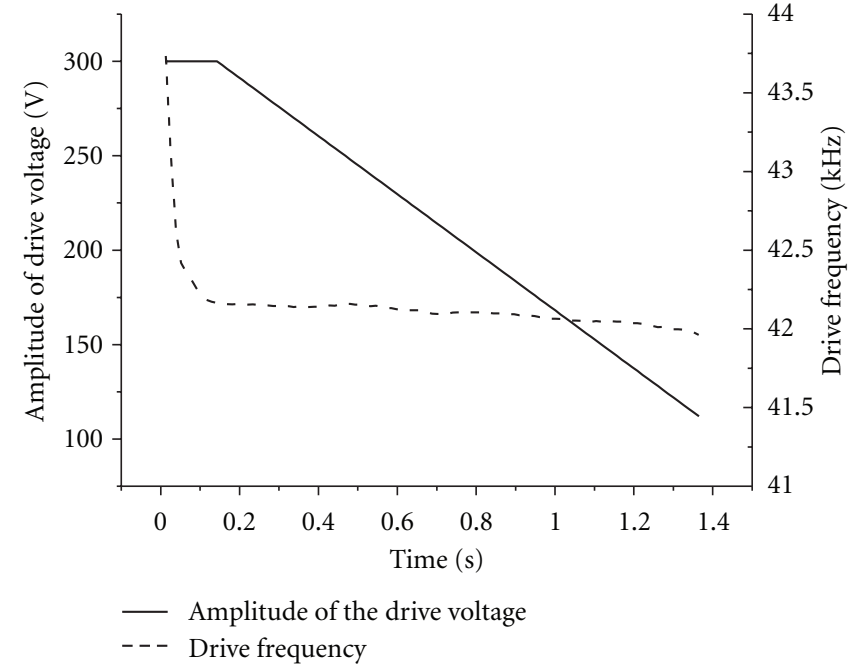

(a) The curve of drive voltage and frequency

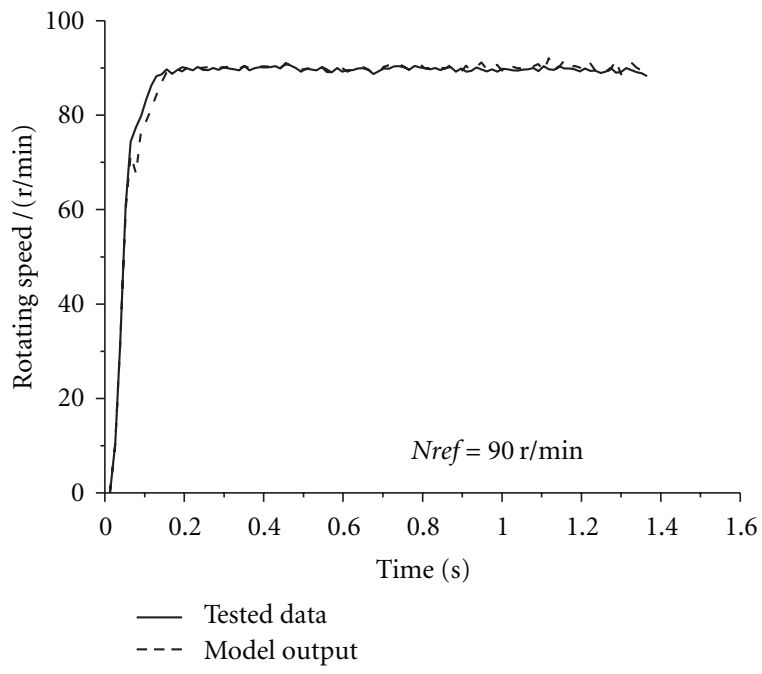

(b) Comparison between model output and tested data

FIGURE 12: Step response comparison between model output and tested data $($ Nre $f=90 \mathrm{r} / \mathrm{min}$, load torque $=0.2 \mathrm{Nm})$.

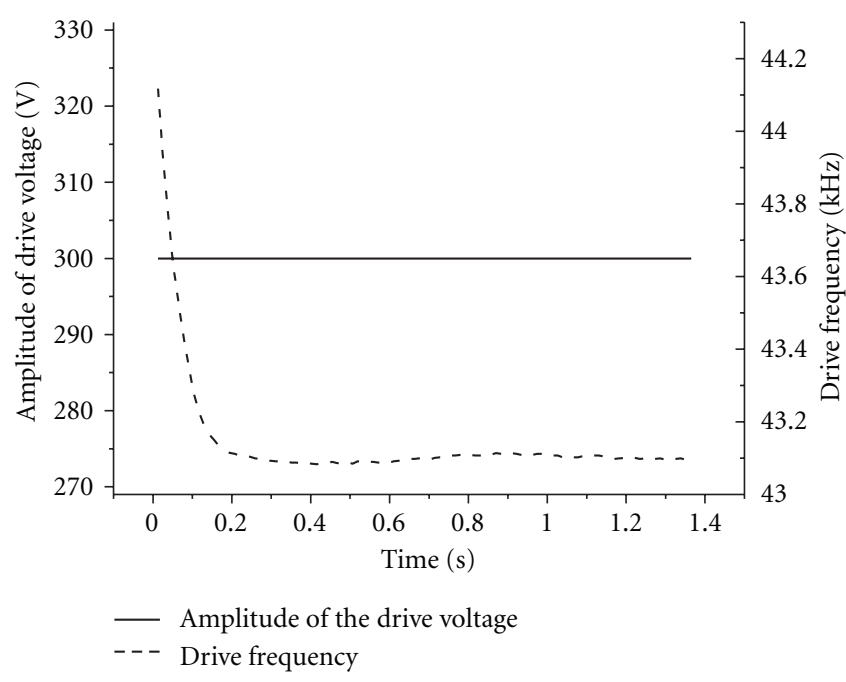

(a) The curve of drive voltage and frequency

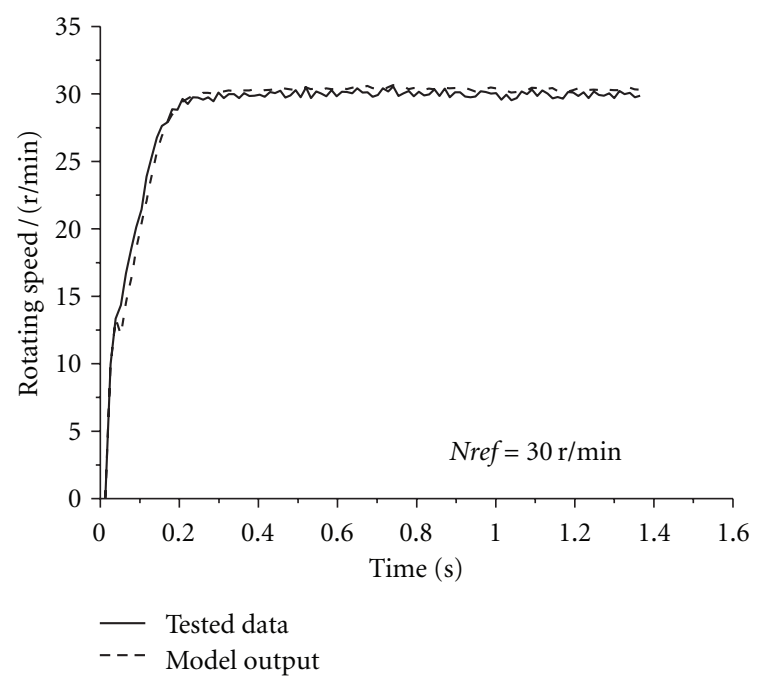

(b) Comparison between model output and tested data

FIGURE 13: Step response comparison between model output and tested data $($ Nref $=30 \mathrm{r} / \mathrm{min}$, Ure $f=300 \mathrm{~V}$, load torque $=0.2 \mathrm{Nm})$.

\section{Dynamic Fuzzy Model for Speed Control of Ultrasonic Motor}

Dynamic fuzzy model reflects the dynamic characteristics. The characteristics of ultrasonic motor system for speed control are related with time. Output variable of the model is the rotating speed value $n(k)$ at the current time. Input variables contain the value of driving voltage amplitude (peak-peak value) $u(k-m)$ and frequency $f(k-m)$ at the current and previous time, where $m=0,1, \ldots$, and also may contain the value of rotating speed $n(k-l)$ at the previous time, where $l=1,2, \ldots$.

The 9 groups of data which are obtained through the above experiments are used for the fuzzy modeling. Among them, the 7 groups are used to model, and the other 2 groups are used as validation data. By studying the tested data, the domain space of $u, f$, and $n$ can be set as $[60,300]$, $[41.9572,45.5482]$ and $[0,120]$, respectively. To simplify the modeling process, the quantification factors of variables are set to 1 , and the quantified variables are defined as $U, F$, and $N$, respectively.

In accordance with the above modeling steps, the process of T-S dynamic fuzzy model is an optimization process. According to the experience and tested data analysis, the value of rotating speed $n(k)$ is related with the value of driving voltage amplitude (peak-peak value) $u(k-m)$, frequency $f(k-m)$ at the current and previous time, and rotating speed $n(k-l)$ at the previous time, where $m=$ $0,1, \ldots$, and $l=1,2, \ldots$ In order to find the proper fuzzy model, $u(k-1), u(k), f(k-1), f(k), n(k-1)$ are firstly 


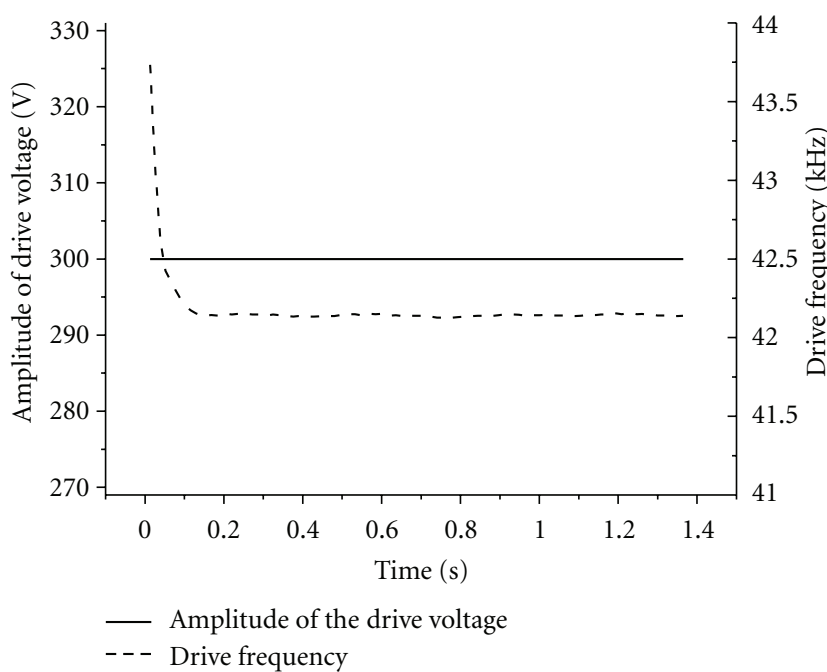

(a) The curve of drive voltage and frequency

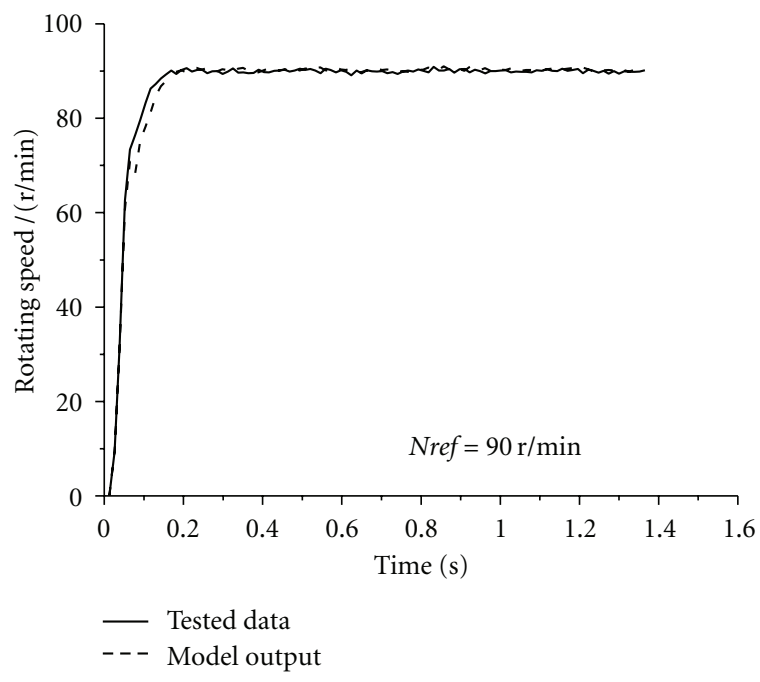

(b) Comparison between model output and tested data

Figure 14: Step response comparison between model output and tested data (Nref $=90 \mathrm{r} / \mathrm{min}$, Ure $f=300 \mathrm{~V}$, load torque $=0.2 \mathrm{Nm})$.

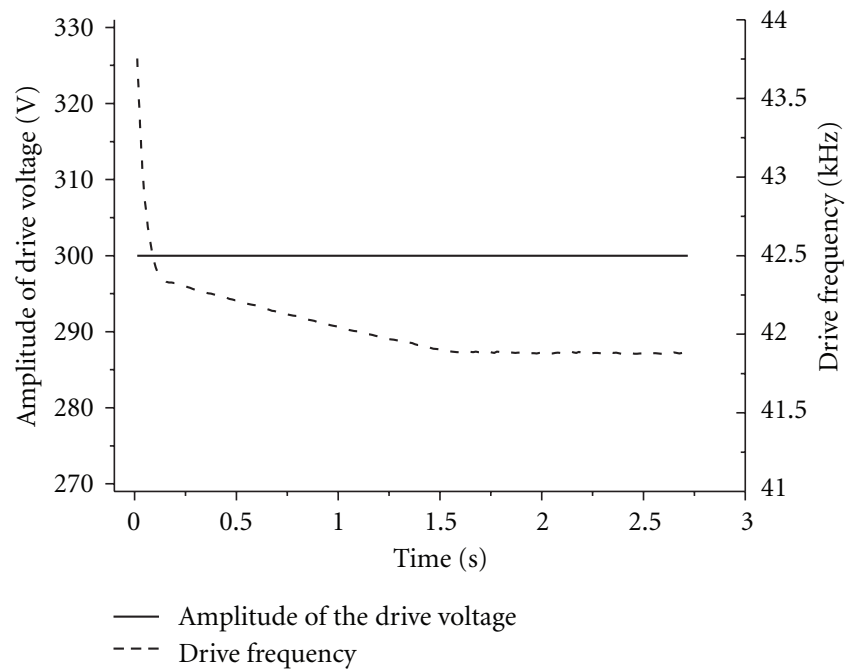

(a) The curve of drive voltage and frequency

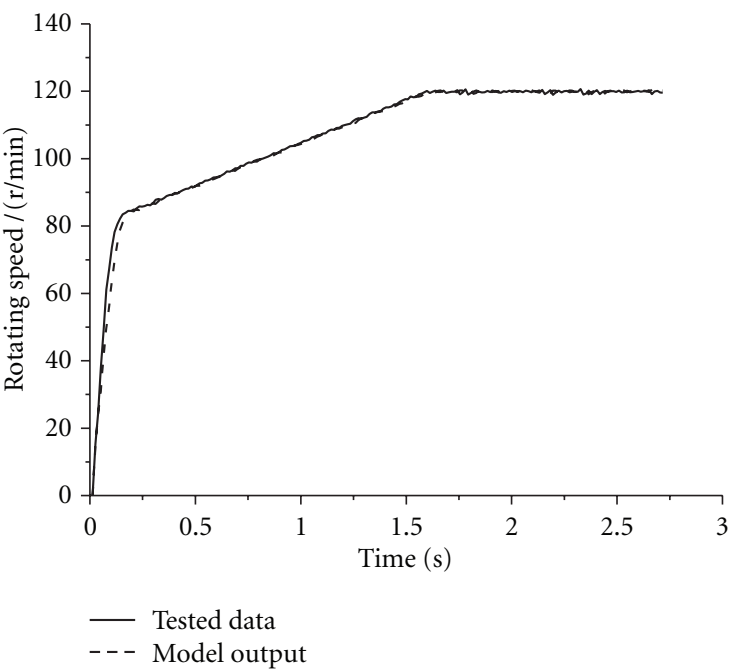

(b) Comparison between model output and tested data

FIGURE 15: Slope response comparison between model output and tested data (Uref $=300 \mathrm{~V}$, no load).

TABLE 2: Fuzzy rules.

\begin{tabular}{|c|c|c|c|c|c|c|}
\hline & $R_{i}$ & $R_{1}$ & $R_{2}$ & $R_{3}$ & $R_{4}$ & $R_{5}$ \\
\hline \multirow{5}{*}{ Premise part } & $u(k-1)$ & $A_{1}$ & $A_{2}$ & $A_{3}$ & $A_{4}$ & $A_{5}$ \\
\hline & $u(k)$ & $A_{1}$ & $A_{2}$ & $A_{3}$ & $A_{4}$ & $A_{5}$ \\
\hline & $f(k-1)$ & $B_{1}$ & $B_{2}$ & $B_{3}$ & $B_{4}$ & $B_{5}$ \\
\hline & $f(k)$ & $B_{1}$ & $B_{2}$ & $B_{3}$ & $B_{4}$ & $B_{5}$ \\
\hline & $n(k-1)$ & $C_{1}$ & $C_{2}$ & $C_{3}$ & $C_{4}$ & $C_{5}$ \\
\hline \multirow{6}{*}{ Conclusion part } & $a_{0}$ & 654.5 & 386.7 & 187.0 & -40.24 & 7.896 \\
\hline & $a_{1}$ & -0.0298 & -0.1323 & -0.0321 & 0.0062 & -0.0855 \\
\hline & $a_{2}$ & 0.0535 & 0.1561 & 0.0522 & -0.0274 & 0.1099 \\
\hline & $a_{3}$ & 38.94 & 29.29 & 5.856 & 9.296 & -0.8417 \\
\hline & $a_{4}$ & -54.12 & -38.35 & -10.23 & -8.257 & 0.5271 \\
\hline & $a_{5}$ & 0.7883 & 0.9124 & 0.9308 & 1.011 & 0.9904 \\
\hline
\end{tabular}




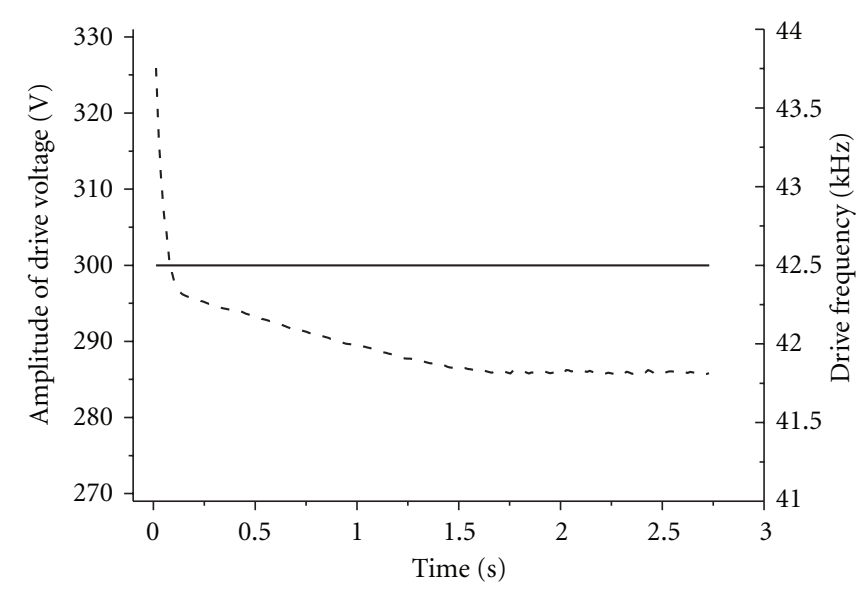

- Amplitude of the drive voltage

- - Drive frequency

(a) The curve of drive voltage and frequency

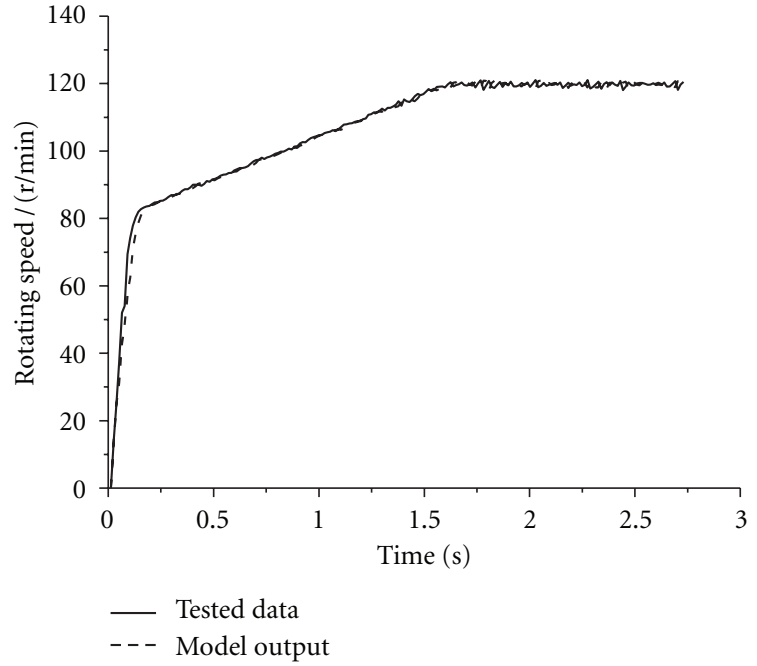

(b) Comparison between model output and tested data

FIGURE 16: Slope response comparison between model output and tested data ( Ure $f=300 \mathrm{~V}$, load torque $=0.2 \mathrm{Nm}$ ).

TABLE 3: Final fuzzy rules.

\begin{tabular}{|c|c|c|c|c|c|c|c|c|c|c|c|}
\hline & $R_{i}$ & $R_{1}$ & $R_{2}$ & $R_{3}$ & $R_{4}$ & $R_{5}$ & $R_{6}$ & $R_{7}$ & $R_{8}$ & $R_{9}$ & $R_{10}$ \\
\hline \multirow{10}{*}{ Premise part } & $u(k-3)$ & $A_{1}$ & $A_{2}$ & $A_{3}$ & $A_{4}$ & $A_{5}$ & $A_{6}$ & $A_{7}$ & $A_{8}$ & $A_{9}$ & $A_{10}$ \\
\hline & $u(k-2)$ & $A_{1}$ & $A_{2}$ & $A_{3}$ & $A_{4}$ & $A_{5}$ & $A_{6}$ & $A_{7}$ & $A_{8}$ & $A_{9}$ & $A_{10}$ \\
\hline & $u(k-1)$ & $A_{1}$ & $A_{2}$ & $A_{3}$ & $A_{4}$ & $A_{5}$ & $A_{6}$ & $A_{7}$ & $A_{8}$ & $A_{9}$ & $A_{10}$ \\
\hline & $u(k)$ & $A_{1}$ & $A_{2}$ & $A_{3}$ & $A_{4}$ & $A_{5}$ & $A_{6}$ & $A_{7}$ & $A_{8}$ & $A_{9}$ & $A_{10}$ \\
\hline & $f(k-3)$ & $B_{1}$ & $B_{2}$ & $B_{3}$ & $B_{4}$ & $B_{5}$ & $B_{6}$ & $B_{7}$ & $B_{8}$ & $B_{9}$ & $B_{10}$ \\
\hline & $f(k-2)$ & $B_{1}$ & $B_{2}$ & $B_{3}$ & $B_{4}$ & $B_{5}$ & $B_{6}$ & $B_{7}$ & $B_{8}$ & $B_{9}$ & $B_{10}$ \\
\hline & $f(k-1)$ & $B_{1}$ & $B_{2}$ & $B_{3}$ & $B_{4}$ & $B_{5}$ & $B_{6}$ & $B_{7}$ & $B_{8}$ & $B_{9}$ & $B_{10}$ \\
\hline & $f(k)$ & $B_{1}$ & $B_{2}$ & $B_{3}$ & $B_{4}$ & $B_{5}$ & $B_{6}$ & $B_{7}$ & $B_{8}$ & $B_{9}$ & $B_{10}$ \\
\hline & $n(k-2)$ & $C_{1}$ & $C_{2}$ & $C_{3}$ & $C_{4}$ & $C_{5}$ & $C_{6}$ & $C_{7}$ & $C_{8}$ & $C_{9}$ & $C_{10}$ \\
\hline & $n(k-1)$ & $C_{1}$ & $C_{2}$ & $C_{3}$ & $C_{4}$ & $C_{5}$ & $C_{6}$ & $C_{7}$ & $C_{8}$ & $C_{9}$ & $C_{10}$ \\
\hline \multirow{11}{*}{ Conclusion part } & $a_{0}$ & 180.9 & 1249 & 145.2 & 255.8 & 169.2 & 130.1 & 13.5 & 30.78 & 59.04 & 38.81 \\
\hline & $a_{1}$ & 0.0937 & -0.04148 & 0.2 & 0.1259 & 0.4378 & 0.3607 & 0.1464 & 0.2738 & 0.8669 & 1.252 \\
\hline & $a_{2}$ & -0.517 & -0.009459 & -0.7707 & -0.273 & -1.328 & -1.252 & -0.5266 & -1.185 & -2.115 & -5.656 \\
\hline & $a_{3}$ & 0.6663 & 0.007595 & 0.7137 & 0.1353 & 1.266 & 1.416 & 0.6314 & 1.586 & 1.617 & 7.234 \\
\hline & $a_{4}$ & -0.2366 & 0.08016 & -0.1412 & 0.0179 & -0.38 & -0.5232 & -0.247 & -0.6777 & -0.3582 & -2.806 \\
\hline & $a_{5}$ & 32.89 & 37.2 & 155.9 & 49.56 & 88.89 & 18.67 & 0.8444 & 22.93 & 134.8 & 22.62 \\
\hline & $a_{6}$ & -7.497 & -108.4 & -342.1 & -96.72 & -87.43 & 44.22 & -21.45 & -6.138 & -163.2 & -9.541 \\
\hline & $a_{7}$ & -20.24 & 173.1 & 275.5 & 53.49 & -70.97 & -129.3 & 51.14 & -44.23 & -64.11 & -42.66 \\
\hline & $a_{8}$ & -9.343 & -130.9 & -92.69 & -12.25 & 65.63 & 63.41 & -30.86 & 26.74 & 91.09 & 28.54 \\
\hline & $a_{9}$ & 0.153 & 0.428 & 0.4712 & -0.5714 & -0.1322 & 0.0339 & 0.8306 & 0.3826 & 0.1745 & -0.0896 \\
\hline & $a_{10}$ & 0.7901 & 0.1586 & 0.486 & 1.49 & 1.079 & 0.9205 & 0.1608 & 0.6079 & 0.807 & 1.076 \\
\hline
\end{tabular}

selected as input variables. The output variable is $n(k)$. The number $p$ of input variables is 5 . The number $c$ of rules is 5 . The centers and widths of Gaussian membership functions are obtained by equalized universe method. The membership functions of $U$ are shown in Figure 6.

After parameter identification, the five fuzzy rules are shown in Table 2. In Table 2, the membership functions of input variables and output variables are $\mathrm{A}_{i}, \mathrm{~B}_{i}$, and $\mathrm{C}_{i}$, in the rule $R_{i}$, respectively. The premise section is fuzzy. The conclusion section is an exact value. The execution time to estimate the six parameters of fuzzy rule $R_{1}$ is $0.2498 \mathrm{~s}$. This time is appropriate for real-time control.

The comparisons of model output data and tested data are shown in Figures 7 and 8. Figure 7 is step response curves when the given value of Nref is $30 \mathrm{r} / \mathrm{min}$. The model output and tested data have the same change trend, but the model output is significantly higher than the tested value. The maximum error is $3.76 \mathrm{r} / \mathrm{min}$, and appears at the initial 
stage of step response. In Figure 8, Nref is $90 \mathrm{r} / \mathrm{min}$ and the model error is less than Figure 7. In the rising stage of step response, the model output is slightly lower than the tested data. In steady stage, the output data is in good agreement with tested data. The maximum error is $12.92 \mathrm{r} / \mathrm{min}$ as shown in Figure 8.

The precision of the model is not high enough as shown in Figures 7 and 8. In order to improve the accuracy, the number $p$ of input variables and the number $c$ of clusters can be changed. After several attempts, compare the model output data with the tested data in different value of $p$ and $c$, and choose the best structure of fuzzy model. The ultimately decision is that the input variables are $u(k-3), u(k-$ 2), $u(k-1), u(k), f(k-3), f(k-2), f(k-1), f(k), n(k-$ $2)$, and $n(k-1)$. The output variable is $n(k)$. The number $p$ of input variables is 10 . The number $c$ of rules is 10 . The membership functions of $U$ are obtained by programming as shown in Figure 9. The fuzzy rules are shown in Table 3. The execution time to estimate the eleven parameters of fuzzy rule $R_{1}$ is $0.2549 \mathrm{~s}$. This time is appropriate for real-time control.

The model output data are given in Figures 10 and 11 with no load. The first point is not painted in Figure 11. Compared with Figure 7, the model error is improved obviously. When load torque is $0.2 \mathrm{Nm}$, Figure 12 shows the speed response as the speed reference is $90 \mathrm{r} / \mathrm{min}$. There is small error in the rising stage. Figures 13 and 14 show the speed response when load torque is $0.2 \mathrm{Nm}$ and Uref is $300 \mathrm{~V}$. The speed response of Figure 13(b) has fluctuations in steady state. Slope response is shown in Figures 15 and 16. The load torque is $0.2 \mathrm{Nm}$ in Figure 16. The speed responses are in good agreement with the given value in Figures 15(b) and 16(b).

\section{Conclusions}

Based on the experimental data, the two-input and oneoutput dynamic model for speed control of ultrasonic motor is worked out using dynamic fuzzy modeling method. The model structure and parameters are identified using equalized universe method and the least-square method, respectively. Using the proposed modeling methods, a few fuzzy rules are needed. The structure of model is simpler. The amount of online calculation is smaller. Comparison between model output and tested data shows that the model can well simulate the nonlinear relationship among the amplitude of driving voltage, frequency, and rotating speed.

This work shows that the fuzzy modeling method is simple. Besides the tested data, membership functions and fuzzy rules can be obtained without other prior knowledge. Fuzzy model has a strong ability of approximating to the nonlinear characteristics and is suitable for ultrasonic motor which is a strong nonlinear system.

\section{References}

[1] W. Chen, S. Shi, Y. Liu, and P. Li, "A new traveling wave ultrasonic motor using thick ring stator with nested PZT excitation," IEEE Transactions on Ultrasonics, Ferroelectrics, and Frequency Control, vol. 57, no. 5, pp. 1160-1168, 2010.

[2] B. Radi and A. El Hami, "The study of the dynamic contact in ultrasonic motor," Applied Mathematical Modelling, vol. 34, no. 12 , pp. 3767-3777, 2010.

[3] Y. Jiajia and R. Xinbo, "Electrical equivalent circuit model of traveling wave rotary ultrasonic motor," Proceedings of the CSEE, vol. 29, no. 15, pp. 80-87, 2009.

[4] T. Senjyu, M. Nakamura, N. Urasaki, H. Sekine, and T. Funabashi, "Mathematical model of ultrasonic motors for speed control," Electric Power Components and Systems, vol. 36, no. 6, pp. 637-648, 2008.

[5] N. Bigdeli and M. Haeri, "Simplified modeling and generalized predictive position control of an ultrasonic motor," ISA Transactions, vol. 44, no. 2, pp. 273-282, 2005.

[6] S. W. Chung and K. T. Chau, "Speed control of travelingwave ultrasonic motors using a practical modeling approach," Electric Power Components and Systems, vol. 35, no. 4, pp. 411428, 2007.

[7] H. Mojallali, R. Amini, R. Izadi-Zamanabadi, and A. A. Jalali, "Systematic experimental based modeling of a rotary piezoelectric ultrasonic motor," ISA Transactions, vol. 46, no. 1, pp. 31-40, 2007.

[8] S. Mou and M. Ouyang, "Establishing the dynamic transfer function of a shaft-driving type ultrasonic motor by system identification," in Proceedings of the 5th World Congress on Intelligent Control and Automation, pp. 302-305, Seoul, Republic of Korea, 2004.

[9] T. C. Chen and C. H. Yu, "Generalized regression neuralnetwork-based modeling approach for traveling-wave ultrasonic motors," Electric Power Components and Systems, vol. 37, no. 6, pp. 645-657, 2009.

[10] F.-J. Lin, R.-J. Wai, and C.-M. Hong, "Identification and control of rotary traveling-wave type ultrasonic motor using neural networks," IEEE Transactions on Control Systems Technology, vol. 9, no. 4, pp. 672-680, 2001.

[11] M. Jahani and H. Mojallali, "Neural network based modeling of traveling wave ultrasonic motor using genetic algorithm," in Proceedings of the 2nd International Conference on Computer and Automation Engineering (ICCAE'10), pp. 486-490, February 2010.

[12] T. C. Chen, C. H. Yu, C. J. Chen, and M. C. Tsai, "Neuro-fuzzy speed control of traveling-wave type ultrasonic motor drive using frequency and phase modulation," ISA Transactions, vol. 47, no. 3, pp. 325-338, 2008.

[13] T. Yoshida, T. Senjyu, M. Nakamura, N. Urasaki, H. Sekine, and T. Funabashi, "Position control of ultrasonic motors using dead-zone compensation with fuzzy neural network," Electric Power Components and Systems, vol. 34, no. 11, pp. 1253-1266, 2006.

[14] F.-J. Lin, S.-Y. Chen, P.-H. Chou, and P. H. Shieh, "Interval type-2 fuzzy neural network control for X-Y-Theta motion control stage using linear ultrasonic motors," Neurocomputing, vol. 72, no. 4-6, pp. 1138-1151, 2009.

[15] F. Bazrafshan, B. Rasti, and H. Mojallali, "Fuzzy modeling and position control of a traveling wave ultrasonic motor," in Proceedings of the 2nd International Conference on Computer and Automation Engineering (ICCAE '10), pp. 457-461, February 2010. 

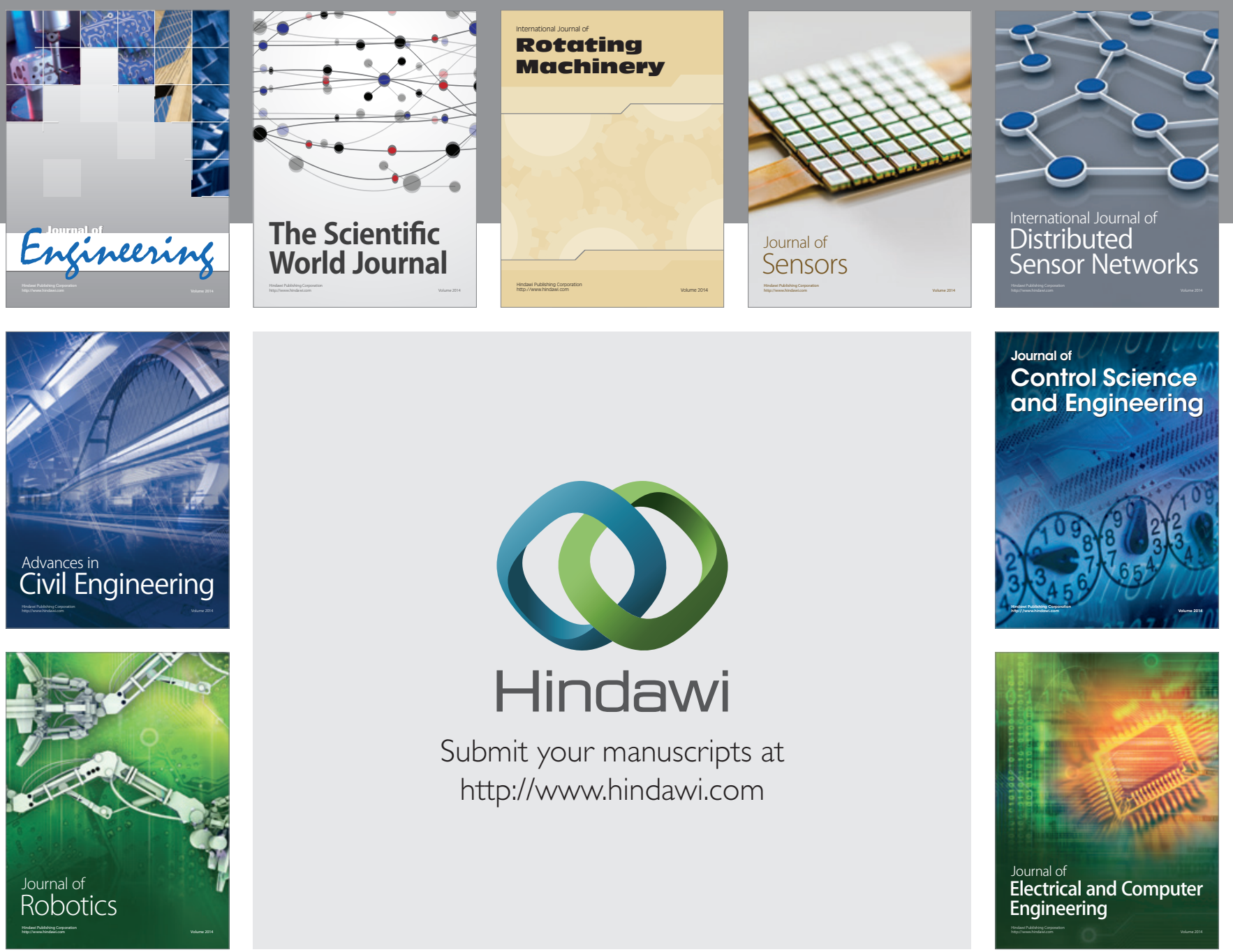

Submit your manuscripts at

http://www.hindawi.com
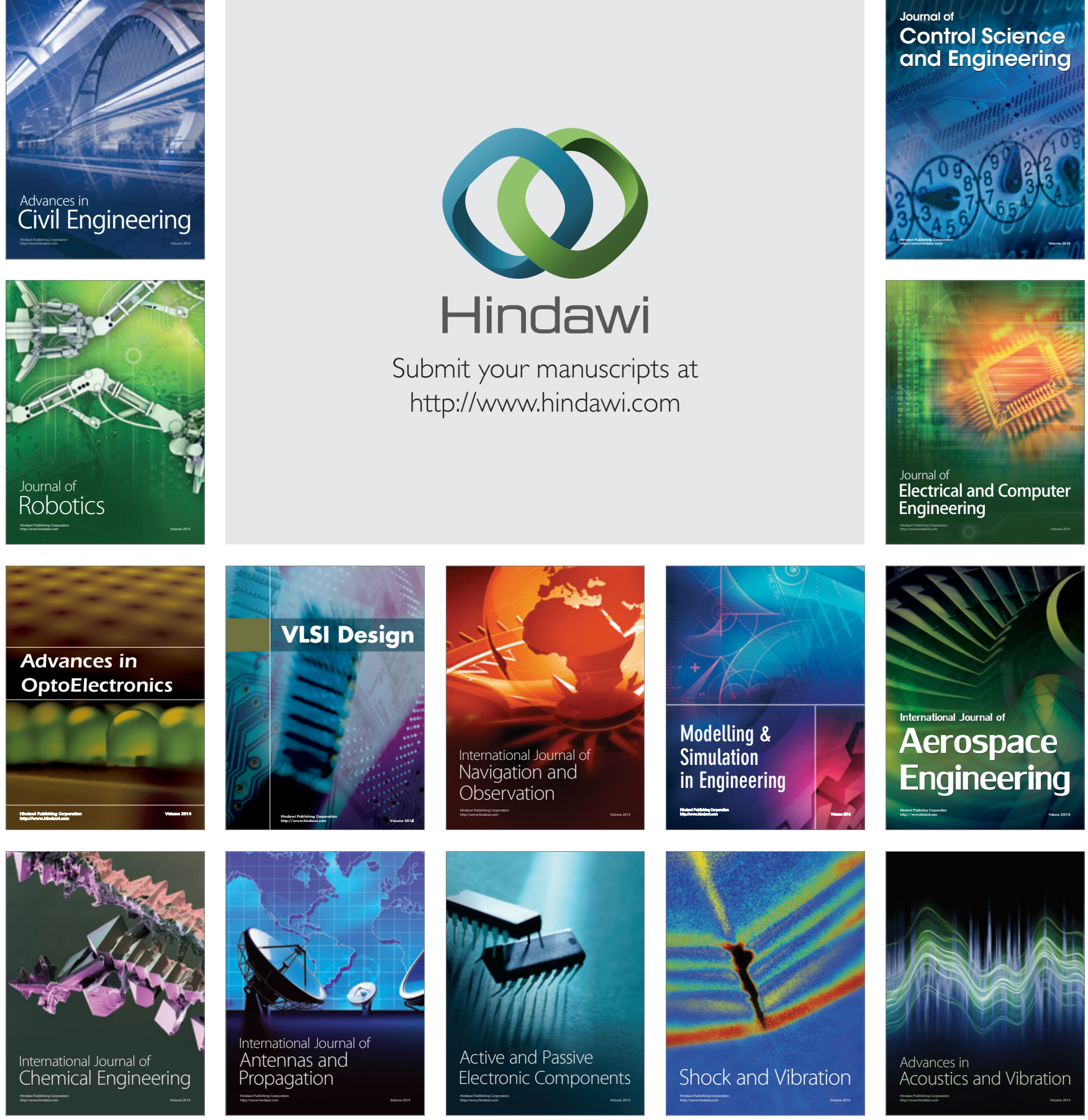